\title{
Spin Hall effect by surface roughness
}

\author{
Lingjun Zhou, ${ }^{1}$ Vahram L. Grigoryan, ${ }^{1}$ Sadamichi Maekawa, ${ }^{2,3}$ Xuhui Wang, ${ }^{4,}$ and Jiang Xiao $(\text { 萧江 })^{1,5, \dagger}$ \\ ${ }^{1}$ Department of Physics and State Key Laboratory of Surface Physics, Fudan University, Shanghai 200433, China \\ ${ }^{2}$ Advanced Science Research Center, Japan Atomic Energy Agency, Tokai 319-1195, Japan \\ ${ }^{3}$ CREST, Japan Science and Technology Agency, Tokyo 102-0075, Japan \\ ${ }^{4}$ King Abdullah University of Science and Technology (KAUST), Physical Science and Engineering Division, \\ Thuwal 23955-6900, Saudi Arabia \\ ${ }^{5}$ Collaborative Innovation Center of Advanced Microstructures, Nanjing University, Nanjing, 210093, China
}

(Received 11 July 2014; revised manuscript received 15 November 2014; published 8 January 2015)

\begin{abstract}
The spin Hall and its inverse effects, driven by the spin orbit interaction, provide an interconversion mechanism between spin and charge currents. Since the spin Hall effect generates and manipulates spin current electrically, to achieve a large effect is becoming an important topic in both academia and industries. So far, materials with heavy elements carrying a strong spin orbit interaction, provide the only option. We propose here a new mechanism, using the surface roughness in ultrathin films, to enhance the spin Hall effect without heavy elements. Our analysis based on $\mathrm{Cu}$ and $\mathrm{Al}$ thin films suggests that surface roughness is capable of driving a spin Hall angle that is comparable to that in bulk $\mathrm{Au}$. We also demonstrate that the spin Hall effect induced by surface roughness subscribes only to the side-jump contribution but not the skew scattering. The paradigm proposed in this paper provides the second, not if only, alternative to generate a sizable spin Hall effect.
\end{abstract}

DOI: 10.1103/PhysRevB.91.045407

PACS number(s): 85.75.-d, 68.35.Ct, 73.50.Jt

\section{INTRODUCTION}

The spin Hall effect, driven by the spin orbit interaction, converts a charge current into a pure spin current [1-11]. Its inverse effect [12], the inverse spin Hall effect, does exactly the opposite. These two effects make it possible to generate and detect spin current electrically. Spin Hall effect and its inverse often find themselves in various applications such as magnetization switching, domain wall motion, spin current detection, etc. All these applications require a highly efficient spin-to-charge interconversion and a large spin Hall effect is thus desired.

In terms of microscopic processes, the spin Hall effect is usually attributed to the intrinsic and extrinsic mechanisms. The intrinsic mechanism originates [1,5,7,13-17] from the band structure properties yet the extrinsic one arises due to the impurity scattering. The extrinsic mechanism further consists of two dominating processes, namely, side jump and skew scattering $[2,11,16,18-22]$, where the former comes from lateral displacement of the wave function during the scattering event and the latter emerges from the spin-dependent scattering cross section. Both extrinsic and intrinsic effects desire a large spin orbit interaction, making heavy atoms the plausible yet only candidate up to date. This explains why the spin Hall phenomena are typically linked with materials consisting of heavy elements, such as $\mathrm{Au}, \mathrm{Pt}$, and Ta.

Many attempts were made towards using different elements or compositions to achieve large spin Hall effect. It is, however, often helpful to explore other possibilities of creating spin Hall effect beyond simply trying one heavy element after another. At this front, a recent proposal on intrinsic spin Hall effect makes use of the interfacial Rashba spin-obit coupling induced by inversion asymmetry at the surface of normal metal thin films [23,24]. In Ref. [23], the

\footnotetext{
*Corresponding author: xuhui.wang@kaust.edu.sa

†Corresponding author: xiaojiang@ fudan.edu.cn
}

spin orbit coupling is generated by the potential gradient normal to the flat surface. In the present paper, as shown in the following sections, the spin-orbit coupling is driven by the potential gradient in the lateral directions.

We propose here a new strategy to accommodate extrinsic spin Hall effect by using the scattering from a rough surface in metallic thin films. The rough surface can be regarded as effective impurities. The finite thickness of the thin film and thus the confinement leads to, in the transverse direction, discrete energy levels that depend on the film thickness. At locations where the thickness is thinner (thicker) than the average thickness, the confinement can be regarded as a repulsive (attractive) potential hill (valley). The electrons transporting inside the film are thus scattered constantly in this random potential landscape and the spin orbit interaction associated with it gives rise to the spin Hall effect.

In our theoretical models, the surface roughness is treated by a dilation operator that transforms a rough surface into an effective surface-impurity potential $[25,26]$. By introducing the spin orbit interaction associated with such effective impurities, we find that even a surface with white noise nature can lead to a side jump contribution to the spin Hall effect. However, interestingly, the skew scattering contribution is absent. The spin Hall angle in a $\mathrm{Cu}$ film is found to be largely comparable to that in bulk Au. Moreover, the film thickness dependence of the spin Hall angle induced by the surface roughness is opposite to that in the bulk spin orbit materials. This unique feature makes it possible to experimentally identify the surface roughness origin of the spin Hall effect.

This paper is organized as follows. In Sec. II, we describe the model for the thin film with surface roughness and the bulk impurities. We put an emphasis on the relaxation times due to bulk impurities and surface impurities, the side jump and skew scattering mechanisms. Section III contributes to the longitudinal and spin Hall conductivities due to the surface scatterings. We also analyze the spin Hall angle due to surface roughness. In Sec. IV, we compare the surface 


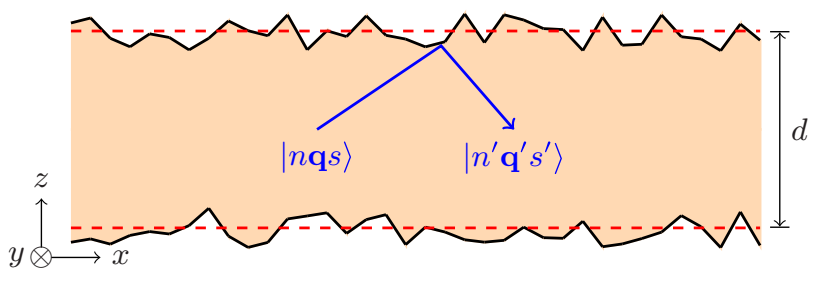

FIG. 1. (Color online) A metallic thin film with rough surfaces, the film thickness at $\rho=(x, y)$ is $d(\rho)$ with $\langle d(\rho)\rangle=d$.

roughness induced spin Hall effect to the one generated by bulk impurities, and discuss the routes to enhance the surface roughness induced spin orbit interaction.

\section{MODEL}

We consider a normal metal thin film with rough surfaces, see Fig. 1 . The film is confined in the $z$ direction and extends in the $\rho=(x, y)$ direction. The film thickness is position dependent $d(\rho)$ with an average $\langle d(\rho)\rangle=d$, given $\langle\cdots\rangle$ the ensemble average. We let $\mathbf{p}_{\|}$and $p_{z}$ be the momentum operator in the $\rho$ and $z$ directions, and then the Hamiltonian is

$$
H=\frac{\mathbf{p}_{\|}^{2}}{2 m^{*}}+\left[\frac{p_{z}^{2}}{2 m^{*}}+V_{d(\rho)}(z)\right]=H_{\|}+H_{\perp}^{d(\rho)},
$$

where $H_{\perp}^{d(\rho)}$ describes the confined quantum well states in the $z$ direction, $m^{*}$ is the electron effective mass, and $V_{d(\rho)}(z)$ is the confining potential with the variable length scale $d(\rho)$. The most convenient way of handling surface roughness is to use the dilation operator. When $d(\rho)$ only slightly deviates from its average value $d$, the dilation operator $[25,26]$

$$
U=e^{\lambda_{\rho}} e^{\lambda_{\rho}\left(z \partial_{z}+\partial_{z} z\right) / 2}
$$

with $\lambda_{\rho} \equiv \ln [d / d(\rho)]$ dilates $H_{\perp}^{d}$ for a quantum well with constant thickness $d$ into $H_{\perp}^{d(\rho)}$ for a well with variable thickness:

$$
H_{\perp}^{d(\rho)}=U H_{\perp}^{d} U^{\dagger}=H_{\perp}^{d}+V_{R}
$$

where

$$
V_{R}=\lambda_{\rho}\left(2 H_{\perp}^{d}+\left[\frac{z \partial_{z}+\partial_{z} z}{2}, H_{\perp}^{d}\right]\right)=\lambda_{\rho}\left(2 V_{d}+z \partial_{z} V_{d}\right)
$$

summarizes the surface roughness into an effective scattering potential that is to be treated perturbatively. Since $d$ is the average thickness, $\left\langle\lambda_{\rho}\right\rangle=0$. For simplicity, we consider a "white noise" surface profile, i.e., the surface roughness is uncorrelated and characterized by the dimensionless parameter $\Lambda \sim(\delta / d)^{2}$ with the thickness deviation variance $\delta^{2}$, so the correlation $[25,26]$

$$
\left\langle\lambda_{\rho} \lambda_{\rho^{\prime}}\right\rangle=\Lambda a^{2} \delta\left(\rho-\rho^{\prime}\right)
$$

with the lattice constant $a \sim k_{F}^{-1}$.

We further introduce two new terms: first, the potential due to the bulk impurities,

$$
V_{I}=\frac{V_{\text {imp }}}{k_{F}^{3}} \sum_{i} \delta\left(\rho-\rho_{i}\right) \delta\left(z-z_{i}\right),
$$

where $\left(\boldsymbol{\rho}_{i}, z_{i}\right)$ is the position of impurity- $i$ and $V_{\mathrm{imp}}$ is the magnitude of the $\delta$-like impurity potential, and, second, the spin orbit interaction due to the surface scattering potential $V_{R}$,

$$
V_{R}^{\mathrm{SO}}=-\eta \hat{\boldsymbol{\sigma}} \cdot\left(\nabla V_{R} \times i \nabla\right) .
$$

In the above, $\hat{\sigma}=\left(\hat{\sigma}_{x}, \hat{\sigma}_{y}, \hat{\sigma}_{z}\right)$ is the Pauli matrix vector and $\eta$ is the spin orbit coupling parameter for the surface scattering. To clarify the role of the surface roughness, the spin orbit coupling due to the bulk impurity $V_{I}$ is neglected for the moment, as we concentrate on the films using metals, such as $\mathrm{Cu}$ or $\mathrm{Al}$, having very weak bulk spin orbit coupling. The full Hamiltonian now becomes

$$
H=H_{0}+U \text { with } U=V_{R}+V_{I}+V_{R}^{\text {SO }},
$$

where $H_{0}=\mathbf{p}^{2} / 2 m^{*}+V_{d}(z)$ describes a film of constant thickness $d$ and $U$ is treated perturbatively.

Without the loss of generality, we assume that the confining potential $V_{d}(z)$ takes a particle-in-box potential, and the eigenstates of $H_{0}$ for a thin film of thickness $d$ are thus given by

$$
E_{n \mathbf{q}}=\frac{\hbar^{2}\left(q^{2}+k_{n}^{2}\right)}{2 m^{*}}, \quad|n \mathbf{q}\rangle=\sqrt{\frac{2}{A d}} \sin \left(k_{n} z\right) e^{i \mathbf{q} \cdot \rho},
$$

where $k_{n}=n \pi / d$ with $n$ labeling the transverse mode, $\mathbf{q}$ is the in-plane wave-vector, and $A$ is the area in the lateral direction. Due to the scattering potential from the bulk impurities $V_{I}$ and surface roughness $V_{R}$, the $|n \mathbf{q}\rangle$ state is mixed with other $\left|n^{\prime} \mathbf{q}^{\prime}\right\rangle$ states. By the Born approximation, the scattered state reads

$$
\left|n \mathbf{q}^{+}\right\rangle=|n \mathbf{q}\rangle+\sum_{n^{\prime} \mathbf{q}^{\prime}}\left|n^{\prime} \mathbf{q}^{\prime}\right\rangle \frac{\left\langle n^{\prime} \mathbf{q}^{\prime}\left|V_{R}+V_{I}\right| n \mathbf{q}\right\rangle}{E_{n \mathbf{q}}-E_{n^{\prime} \mathbf{q}^{\prime}}+i \epsilon} .
$$

Taking into the spin part of the wave function, the scattered state is becoming $\left|n \mathbf{q} s^{+}\right\rangle \equiv\left|n \mathbf{q}^{+}\right\rangle|s\rangle$ and $|s\rangle$ is the eigenspin state with $\hat{\sigma}_{z}|s\rangle=s|s\rangle$. The matrix element $\left\langle n^{\prime} \mathbf{q}^{\prime}\left|V_{R}\right| n \mathbf{q}\right\rangle \approx$ $\lambda_{\mathbf{q}-\mathbf{q}^{\prime}} E_{0} n n^{\prime}\left[(-1)^{n+n^{\prime}}+1\right]$ with $E_{0}=\hbar^{2} \pi^{2} / 2 m d^{2}$, where $\lambda_{\mathbf{q}-\mathbf{q}^{\prime}}$ is the Fourier component of $\lambda_{\rho}$ of the wave vector $\mathbf{q}-\mathbf{q}^{\prime}$.

\section{A. Relaxation times}

The electron relaxation time, or the inverse of scattering rate, can be calculated from the transition probability

$$
P_{n^{\prime} \mathbf{q}^{\prime} s^{\prime}}^{n \mathbf{q} s}=\frac{2 \pi}{\hbar}\left|\left\langle\left\langle n^{\prime} \mathbf{q}^{\prime} s^{\prime}|T| n \mathbf{q} s\right\rangle\right\rangle\right|^{2} \delta\left(E_{n^{\prime} \mathbf{q}^{\prime} s^{\prime}}-E_{n \mathbf{q} s}\right),
$$

where $T=U+U(E-H)^{-1} U$. The double $\langle\langle\cdots\rangle\rangle$ denotes the expectation value average over the scattered state $\left|n \mathbf{q} s^{+}\right\rangle$ and the ensemble average over roughness profiles (and/or impurity distributions). We consider here that the surface roughness and the impurity distribution are uncorrelated. To the leading order in $\Lambda$ and $V_{\text {imp }}$, the total scattering rate, satisfying the Matthiessen's rule, is the sum of the rates due to both the surface and impurity scatterings [25,26]:

$$
\frac{1}{\tau_{n}}=\sum_{n^{\prime} \mathbf{q}^{\prime} s^{\prime}} P_{n^{\prime} \mathbf{q}^{\prime} s^{\prime}}^{n \mathbf{q} s}=\frac{1}{\tau_{0}}+\frac{1}{\tau_{n}^{\prime}}=\frac{1}{\tau_{0}}+\frac{n^{2}}{\tau^{\prime}} .
$$


Here, $1 / \tau_{0}$ is the bulk impurity scattering rate and $1 / \tau_{n}^{\prime}=$ $n^{2} / \tau^{\prime}$ is the channel-dependent surface scattering rate:

$$
\begin{aligned}
\frac{1}{\tau_{0}} & =\left(1+\frac{1}{2 n_{c}}\right) \frac{n_{i}}{2 \pi k_{F}^{3}} \frac{V_{\mathrm{imp}}^{2}}{E_{F}^{2}} \frac{E_{F}}{\hbar}, \\
\frac{1}{\tau^{\prime}} & =\frac{\delta^{2}}{a^{2}} \frac{4 S}{3 n_{c}^{3}} \frac{E_{F}}{\hbar},
\end{aligned}
$$

where $n_{c}=\left\lfloor k_{F} d / \pi\right\rfloor$ is the total number of transverse channels and $S=3 \sum_{n^{\prime}=1}^{n_{c}} n^{\prime 2} / n_{c}^{3} \simeq 1$. The surface scattering rate increases with $n$ and this is because the electrons with larger $n$ are more energetic in the transverse direction, bouncing to the surface more frequently and being scattered more often. We distinguish two limiting cases in the following: (i) $\tau^{\prime} \ll \tau_{0}$, where the surface scattering scattering rules the electron relaxation, and (ii) $\tau^{\prime} \gg n_{c}^{2} \tau_{0}$, where the bulk impurity scattering dominates.

\section{B. Side jump}

The in-plane velocity operator is calculated from the Hamiltonian (7) as [23]

$$
\hat{\mathbf{v}}_{\|}=-\frac{i}{\hbar}[\rho, H]=\frac{\hat{\mathbf{p}}_{\|}}{m^{*}}+\frac{\eta}{\hbar} \hat{\sigma}_{z} \hat{\mathbf{z}} \times \nabla_{\|} V_{R},
$$

where the second term is the anomalous velocity due to the surface scattering and $\nabla_{\|}$is the gradient in the in-plane $\rho$ direction. We only retain the $\nabla_{\|} V_{R}$ component since the gradient in the $z$ direction vanishes after ensemble and state average, i.e., $\left\langle\left\langle\partial_{z} V_{R}\right\rangle\right\rangle=0$.

The charge current, carried by $\left|n \mathbf{q} s^{+}\right\rangle, \quad \mathbf{j}_{n \mathbf{q} s}=$ $\left\langle\left\langle n \mathbf{q} s^{+}\left|e \hat{\mathbf{v}}_{\|}\right| n \mathbf{q} s^{+}\right\rangle\right\rangle$is

$$
\mathbf{j}_{n \mathbf{q} s}=e \frac{\hbar \mathbf{q}}{m^{*}}+e s \alpha_{n}^{\mathrm{sj}} \hat{\mathbf{z}} \times \frac{\hbar \mathbf{q}}{m^{*}} \quad \text { with } \quad \alpha_{n}^{\mathrm{sj}}=\frac{m^{*} \eta}{\hbar \tau_{n}^{\prime}} .
$$

We have introduced $\alpha_{n}^{\mathrm{sj}}$ as the channel-dependent dimensionless coupling parameter of side jump. The first term in Eq. (14) is the normal charge current, and the second term is due to the anomalous velocity giving rise to the spin Hall current. $\alpha_{n}^{\mathrm{sj}}$ depends only on $\tau_{n}^{\prime}$ but not $\tau_{0}$ as we only consider the spin-orbit interaction $V_{R}^{\mathrm{SO}}$ from the surface scattering.

\section{Skew scattering}

Besides the side-jump contribution to the spin Hall current, there is usually also the skew scattering contribution originating from the asymmetric transition probability $P_{n^{\prime} \mathbf{q}^{\prime} s^{\prime}}^{n \mathbf{q} s}$ between $\mathbf{q}$ and $\mathbf{q}^{\prime}$ induced by the spin orbit interaction. Yet in the present case of surface roughness scattering, the asymmetric transition probability vanishes: the asymmetric transition probability involves the three-body averages of $\left\langle\left\langle U^{3}\right\rangle\right\rangle$, with $U=V_{R}+V_{I}+V_{R}^{\mathrm{SO}}$. For asymmetric part, $V_{R}^{\mathrm{SO}}$ must be involved, thus the terms contributing to the asymmetric transition probability include $\left\langle\left\langle V_{I} V_{I} V_{R}^{\mathrm{SO}}\right\rangle\right\rangle,\left\langle\left\langle V_{R} V_{R} V_{R}^{\mathrm{SO}}\right\rangle\right\rangle,\left\langle\left\langle V_{I} V_{R} V_{R}^{\mathrm{SO}}\right\rangle\right\rangle$. The first term $\left\langle\left\langle V_{I} V_{I} V_{R}^{\mathrm{SO}}\right\rangle\right\rangle \propto\left\langle\lambda_{\rho}\right\rangle=0$ because $\lambda_{\rho}$ has a zero average. The second term $\left\langle\left\langle V_{R} V_{R} V_{R}^{\mathrm{SO}}\right\rangle\right\rangle \propto\left\langle\left\langle\lambda_{\mathbf{q}} \lambda_{\mathbf{q}^{\prime}} \lambda_{-\mathbf{q}-\mathbf{q}^{\prime}}\right\rangle\right\rangle=$ 0 , according to the white noise profile of surface roughness considered in this paper. The last $\left\langle\left\langle V_{I} V_{R} V_{R}^{\mathrm{SO}}\right\rangle\right\rangle \propto\left\langle\left\langle V_{I}\right\rangle\right\rangle=0$, when the initial and final states are different, which is the case for the asymmetric transitions: there is no asymmetry when initial and final states are the same. Therefore, the surface roughness induced spin orbit interaction does not lead to any skew scattering contribution and consequently the coupling parameter for skew scattering vanishes: $\alpha^{\text {ss }}=0$.

In a simpler physical picture, the absence of skew scattering is caused by the random sign of the surface roughness potential $V_{R} \propto \lambda_{\rho}$, which is attractive when $\lambda_{\rho}<0$ (or $d(\rho)>d$ ) and becomes repulsive when $\lambda_{\rho}>0($ or $d(\rho)<d)$. Therefore, the ensemble average over the surface roughness of $\left\langle\left\langle V_{R}^{3}\right\rangle\right\rangle$ vanishes due to the random sign. This situation is different in the case of the impurity scattering, in which the sign of the impurity scattering potential is fixed, i.e., the impurities are all either attractive or repulsive.

\section{CONDUCTIVITIES}

\section{A. Longitudinal conductivity}

When an in-plane electric field $\mathbf{E}$ is applied, the Fermi circle shifts $\delta \mathbf{q}_{n}=e \mathbf{E} \tau_{n} / \hbar$ for channel $n$. The corresponding nonequilibrium distribution function is

$$
g_{n \mathbf{q}}=f_{n}\left(\mathbf{q}+\delta \mathbf{q}_{n}\right)-f_{n}(\mathbf{q})=\frac{e \hbar \tau_{n}}{m^{*}} \delta\left(E_{n \mathbf{q}}-E_{F}\right) \mathbf{q} \cdot \mathbf{E},
$$

where $f_{n}(\mathbf{q})=\Theta\left(E_{n \mathbf{q}}-E\right)$ is the Fermi-Dirac distribution function with $\Theta$ the Heaviside function. The normal velocity in Eq. (14) contributes to the in-plane longitudinal charge current $\mathbf{j}=\sum_{n \mathbf{q} s} g_{n \mathbf{q}} \mathbf{j}_{n \mathbf{q} s}=\sigma \mathbf{E}$ with the longitudinal conductivity

$$
\sigma=\frac{3 \sigma_{0}}{2 n_{c}} \sum_{n=1}^{n_{c}} \frac{\tau_{n}}{\tau_{0}}\left(1-\frac{n^{2}}{n_{c}^{2}}\right)
$$

where $\sigma_{0}=\frac{k_{F}^{3}}{3 \pi^{2}} \frac{e^{2} \tau_{0}}{m^{*}}$ is the bulk conductivity in Drude model. In the limit where surface scattering dominates or bulk impurity scattering dominates, we have

$$
\sigma \simeq\left\{\begin{array}{lll}
\frac{e^{2} k_{F}}{2 \hbar}\left(\frac{d}{\delta}\right)^{2} & \text { for } \quad \tau^{\prime} \ll \tau_{0}, \\
\sigma_{0}\left(1-\frac{3}{4 n_{c}}\right) & \text { for } \quad \tau^{\prime} \gg n_{c}^{2} \tau_{0} .
\end{array}\right.
$$

The charge conductivity (16) agrees with Ref. [26].

The film thickness dependence $\left(n_{c} \simeq k_{F} d / \pi\right)$ of the longitudinal conductivity (16) for $\mathrm{Cu}$ film is plotted in the left panel of Fig. 2 for three different levels of roughness: $\delta=$ $0, a, 10 a$. The conductivity increases with increasing thickness, as expected. For a flat surface $(\delta=0)$, the increase of conductivity is simply due to the quantum size effect when the film is thin. For a rough surface $(\delta=a, 10 a)$, the increase in the conductivity is attributed to the decreasing of the surface scattering induced resistivity as the film is becoming thicker. The right panel of Fig. 2 shows the surface roughness $(\delta)$ dependence of the conductivity for three different film thickness $n_{c}=10,100,1000$. For all cases, the conductivity decreases with increasing surface roughness.

Since we treat the surface roughness as perturbation, Eq. (16) is valid only when the roughness is small comparing to the film thickness: $\delta \ll d$. Therefore Eq. (16) is not a good approximation in the shaded area in Fig. 2. 

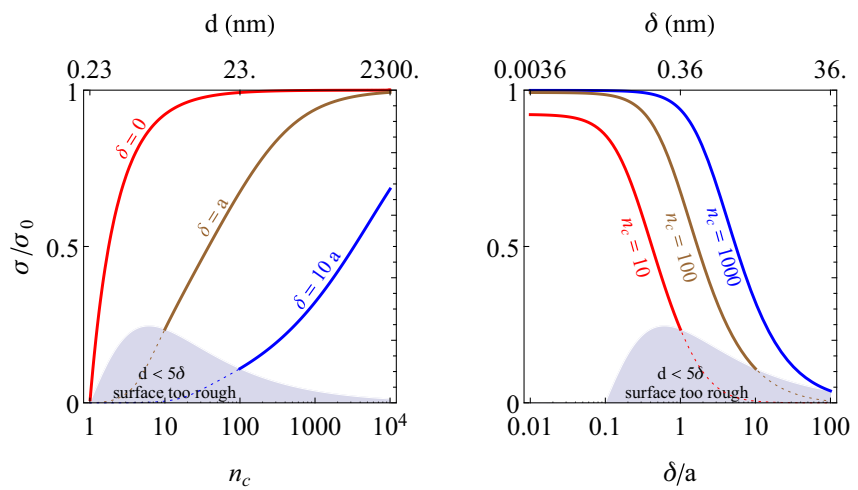

FIG. 2. (Color online) Longitudinal conductivity for $\mathrm{Cu}$ film, $k_{F}=1.36 \times 10^{10} / \mathrm{m}, a=3.61 \AA$, and $\sigma_{0}=5.88 \times 10^{7} \mathrm{~S} / \mathrm{m}\left(\tau_{0} \sim 24\right.$ fs). (Left) $\sigma$ as function of film thickness $n_{c} \simeq k_{F} d / \pi$ for three cases of roughness $\delta=0, a, 10 a$. (Right) $\sigma$ as function of the surface roughness $\delta / a$ for three cases of film thickness $n_{c}=10,100,1000$. In the shaded area, the surface is too rough $(\delta / d>0.2)$ and the perturbation assumption fails.

\section{B. In-plane spin Hall conductivity}

The second anomalous term in Eq. (14) does not lead to any charge current for its opposite spin dependence. However, it gives rise to an in-plane pure spin current in the transverse direction or a spin Hall current $\mathbf{j}_{s}=\sum_{n \mathbf{q} s} s g_{n \mathbf{q}} \mathbf{j}_{n \mathbf{q} s}=\sigma^{\mathrm{sH}} \hat{\mathbf{z}} \times$ E. In the unit of charge current, the spin Hall conductivity reads

$$
\sigma^{\mathrm{sH}}=\frac{e^{2} k_{F}}{h} \frac{\bar{\eta}}{\pi} \sum_{n=1}^{n_{c}} \frac{\tau_{n}}{\tau_{n}^{\prime}}\left(\frac{1}{n_{c}}-\frac{n^{2}}{n_{c}^{3}}\right)
$$

with $\bar{\eta}=\eta k_{F}^{2}$ the dimensionless spin orbit coupling parameter. At the two limits where surface scattering dominates and bulk impurity scattering dominates, the spin Hall conductivity is

$$
\sigma^{\mathrm{sH}} \simeq \frac{e^{2} k_{F}}{h} \frac{\bar{\eta}}{\pi} \begin{cases}\frac{2}{3} & \text { for } \quad \tau^{\prime} \ll \tau_{0}, \\ \frac{2 n_{c}^{2} \tau_{0}}{15 \tau^{\prime}} & \text { for } \quad \tau^{\prime} \gg n_{c}^{2} \tau_{0} .\end{cases}
$$

When the surface roughness dominates, the spin Hall conductivity in Eq. (19) is independent of the surface roughness $\delta$ for the following reason: $\sigma^{\mathrm{sH}}$ is proportional to both the side jump coupling parameter $\alpha_{n}^{\mathrm{sj}} \propto \tau^{\prime-1} \propto \delta^{2}$ and the Fermi circle shift $\delta \mathbf{q}_{n} \propto \tau_{n} \sim \tau^{\prime} \propto \delta^{-2}$, thus the dependence on the relaxation time $\tau^{\prime}$ (or the surface roughness $\delta$ ) cancels. This behavior is the same as the side jump contribution to the spin Hall conductivity in the bulk materials.

Taking $\bar{\eta}=0.5$, that is, similar to the bulk value in Pt [11], the left panel of Fig. 3 plots $\sigma^{\mathrm{sH}}$ as a function of $\delta$, showing that $\sigma^{\mathrm{sH}}$ is larger for a rougher surface and thinner film. In conventional bulk spin Hall effect, the relation between the spin Hall resistivity $\rho^{\mathrm{sH}}=\sigma^{\mathrm{sH}} / \sigma^{2}$ can be expressed in terms of the longitudinal resistivity $\rho=1 / \sigma: \rho^{\mathrm{sH}}=a \rho+b \rho^{2}$, where the linear and quadratic terms are due to the skew scattering and side jump mechanisms, respectively. We show in the right panel of Fig. 3 the log-log plot of $\rho^{\mathrm{sH}}$ as function of $\rho$, which includes the bulk resistivity $\rho_{0}=1 / \sigma_{0}$ and the surface scattering induced resistivity $\rho-\rho_{0}$. In the surface scattering dominating region (blue squares), the slope of a linear fit is 2.1, i.e., $\rho^{\mathrm{sH}} \propto\left(\rho-\rho_{0}\right)^{2.1} \simeq \rho^{2.1}$, consistent with the side jump
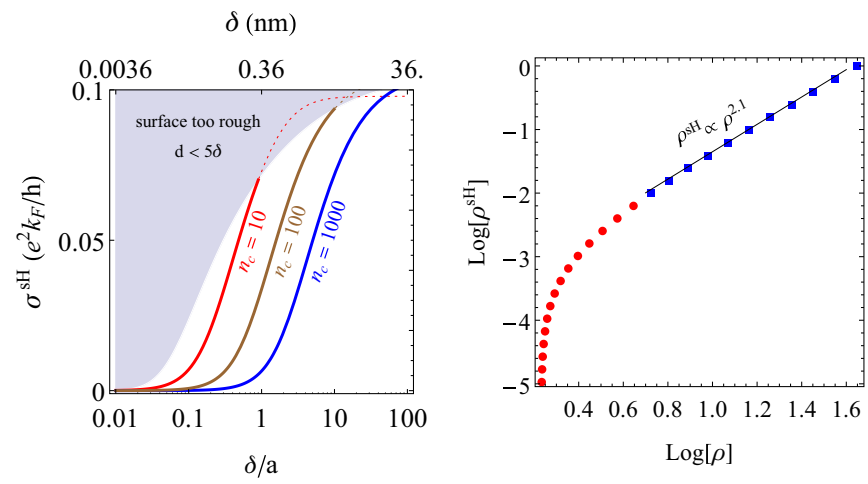

FIG. 3. (Color online) Spin Hall conductivity $\sigma^{\mathrm{sH}}$ for $\mathrm{Cu}$ film with the same parameter as in Fig. 2 and $\bar{\eta}=0.5$. (Left) $\sigma^{\mathrm{sH}}$ as function of surface roughness $\delta / a$. (Right) Spin Hall resistivity $\rho^{\mathrm{sH}}=\sigma^{\mathrm{sH}} / \sigma^{2}$ as a function of resistivity $\rho=1 / \sigma$ (by varying surface roughness $\delta$ ) for $n_{c}=1000$. The linear fit in the surface roughness dominating region (blue squares) has $\rho^{\mathrm{sH}} \propto \rho^{2.1}$, consistent with the side jump mechanism.

mechanism. The slight deviation from slope 2 is due to the mixing of bulk relaxations, as well as the different relaxation time $\tau_{n}$ for different transverse channels.

\section{Spin Hall angle}

With both the longitudinal conductivity (16) and the spin Hall conductivity (18), the spin Hall angle is obtained to be

$$
\theta^{\mathrm{sH}}=\frac{\sigma^{\mathrm{sH}}}{\sigma} \simeq \bar{\eta}\left(\frac{\delta}{d}\right)^{2}\left\{\begin{array}{l}
\frac{1}{3 \pi^{2}} \\
\frac{n_{c}}{30}\left(\frac{2 \pi}{k_{F} a}\right)^{2}
\end{array}\right.
$$

The upper (lower) approximation in Eq. (20) corresponds to the surface (impurity) scattering dominating case with $\tau_{n}^{\prime} \ll \tau_{0}$ $\left(\tau_{n}^{\prime} \gg \tau_{0}\right)$.
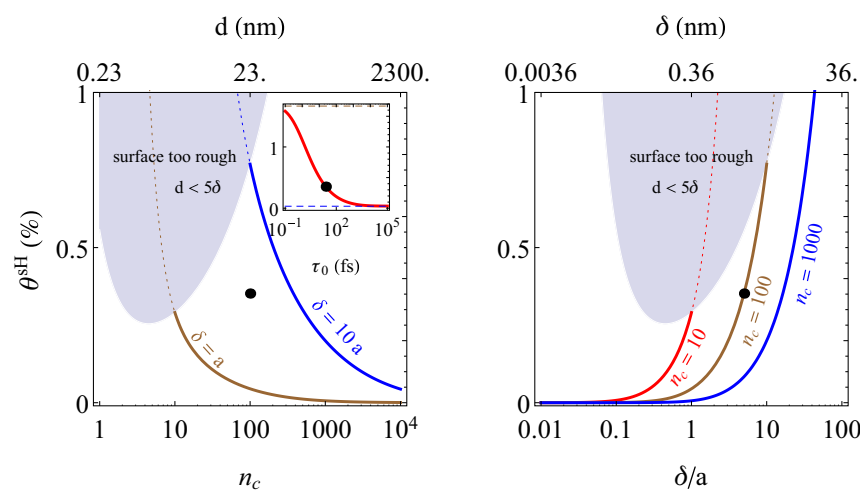

FIG. 4. (Color online) The spin Hall angle (in percent) for $\mathrm{Cu}$ film, with the same parameter as in Fig. 2 and $\bar{\eta}=0.5$, (left) as a function of film thickness $n_{c} \simeq k_{F} d / \pi$. Inset figure shows the $\theta^{\mathrm{sH}}$ dependence on the bulk relaxation time $\tau_{0}$ for $n_{c}=100$ and $\delta=5 a$ with the dashed lines given by the limiting values in Eq. (20) (right) as a function of the surface roughness $\delta / a$. The black dot in all plots corresponds to the same point with $n_{c}=100(d \simeq 23 \mathrm{~nm}), \delta=5 a \simeq$ $1.8 \mathrm{~nm}, \tau_{0} \sim 24$ fs for $\mathrm{Cu}$ with conductivity $\sigma_{0}=5.88 \times 10^{7} \mathrm{~S} / \mathrm{m}$, and has spin Hall angle $\theta^{\mathrm{sH}} \simeq 0.35 \%$. 
TABLE I. Surface roughness induced spin Hall angle for thin films of several normal metals that has no bulk spin Hall effect. In all cases, $n_{c}=100, \delta=5 a, \bar{\eta}=0.5$, and electron effective mass equals to the free electron mass $m^{*}=m$. Data for $\sigma_{0}, k_{F}$ from Refs. [27,28].

\begin{tabular}{lccc}
\hline \hline Material & $\sigma_{0}\left(10^{7} \mathrm{~S} / \mathrm{m}\right)$ & $k_{F}(1 / \AA)$ & $\theta^{\mathrm{sH}}$ \\
\hline $\mathrm{Cu}$ & 5.88 & 1.36 & $0.35 \%$ \\
$\mathrm{Ag}$ & 6.21 & 1.19 & $0.32 \%$ \\
$\mathrm{Au}$ & 4.55 & 1.21 & $0.37 \%$ \\
$\mathrm{Al}$ & 3.65 & 1.75 & $0.48 \%$ \\
\hline \hline
\end{tabular}

As shown in Fig. 4, the spin Hall angle can be enhanced by (i) decreasing film thickness $n_{c}$, (ii) increasing surface roughness $\delta$, (iii) decreasing bulk relaxation time $\left(\tau_{0}\right)$ or increasing bulk resistivity $\left(1 / \sigma_{0}\right)$. For $n_{c}=100$ and $\delta=5 a$, the spin Hall angle for thin films made of $\mathrm{Cu}, \mathrm{Al}$, and $\mathrm{Ag}$ are listed in Table I For $\mathrm{Cu}, d \simeq 23 \mathrm{~nm}$ and $\delta \simeq 1.8 \mathrm{~nm}$, the spin Hall angle for $\mathrm{Cu}$ film can reach a fraction of a percent $(0.35 \%)$, which is comparable to that for bulk Au.

\section{DISCUSSION AND CONCLUSIONS}

We have also carried out the same calculation for a thin film where the spin orbit interaction comes solely from the bulk impurity scattering instead of the surface scattering, i.e., the $V_{\mathrm{R}}^{\mathrm{SO}}$ in $H \mathrm{Eq}$. (7) is replaced by $V_{I}^{\mathrm{SO}}=-\eta_{I} \hat{\sigma} \cdot\left(\nabla V_{I} \times\right.$ $i \nabla)$ with $\eta_{I}$ the spin-orbit interaction parameter for impurity scattering potential $V_{I}$. The full Hamiltonian becomes

$$
H=H_{0}+U^{\prime} \quad \text { with } U^{\prime}=V_{R}+V_{\mathrm{I}}+V_{\mathrm{I}}^{\text {SO }}
$$

Following Takahashi et al. [11], we find the coupling parameter of side jump and skew scattering as [29]

$$
\alpha_{I}^{\mathrm{sj}}=\frac{m^{*} \eta_{I}}{\hbar \tau_{0}} \quad \text { and } \quad \alpha_{I}^{\mathrm{ss}}=\frac{\bar{\eta}_{I}}{12 \pi} \frac{V_{\mathrm{imp}}}{E_{F}} \frac{\sigma}{\sigma_{0}},
$$

where $\bar{\eta}_{I}=\eta_{I} k_{F}^{2}$ and $\sigma$ is the same conductivity as Eq. (16). The spin Hall conductivity $\sigma_{I}^{\mathrm{sH}}=\left(\alpha_{I}^{\mathrm{sj}}+\alpha_{I}^{\mathrm{ss}}\right) \sigma$. Therefore the spin Hall angle for the bulk impurity induced spin Hall effect in metallic thin film is $\theta_{I}^{\mathrm{SH}}=\alpha_{I}^{\mathrm{sj}}+\alpha_{I}^{\mathrm{ss}}$. Since side jump contribution $\alpha_{I}^{\mathrm{sj}}$ is a constant, independent of film thickness and/or surface roughness, and skew scattering contribution $\alpha_{I}^{\text {ss }}$ has the same parameter dependence as the longitudinal conductivity $\sigma$, therefore the spin Hall angle by impurity scattering in metallic thin films decreases with decreasing film thickness and/or increasing surface roughness (following the same trend as $\sigma$ in Fig. 2). This behavior is opposite to that for the spin Hall angle by surface scattering (see Fig. 4). Therefore it is possible to distinguish the origin of the spin Hall effect from the thickness and/or surface roughness dependence of the spin Hall angle.

To realize the surface roughness induced spin Hall effect experimentally, it is necessary to have two crucial ingredients simultaneously: (i) the surface roughness, which acts as surface impurities, (ii) the strong interfacial (not bulk) spin-orbit interaction. The former can be controlled by thin film growing process or the surface polishing technique. The latter is made possible by coating the metallic thin film surface with materials with large spin orbit interaction, such as Pt or oxides with heavy elements. In such a way, one may utilize the strong scattering due to roughness and the strong spin-orbit interaction from the heavy elements.

In conclusion, we predict that, even in metallic thin films without bulk spin-orbit interaction, the spin Hall effect can be realized by surface roughness. The surface roughness induced spin Hall effect comprises only side jump contribution. This new approach makes it possible to realize spin Hall effect in the normal metals like $\mathrm{Cu}, \mathrm{Al}$ without using the noble metals. We predict that for $\mathrm{Cu}$ film with sizable interfacial spin-orbit interaction, the spin Hall angle can be as large as $0.35 \%$, which is comparable to that in $\mathrm{Au}$. The spin Hall angle due to surface scattering increases with reducing the film thickness, opposite to that due to the bulk impurity scattering, and its existence can thus be experimentally identified.

\section{ACKNOWLEDGMENTS}

This work was supported by the special funds for the Major State Basic Research Project of China (2014CB921600, 2011CB925601) and the National Natural Science Foundation of China (91121002).
[1] R. Karplus and J. M. Luttinger, Phys. Rev. 95, 1154 (1954).

[2] M. Dyakonov and V. Perel, Phys. Lett. A 35, 459 (1971).

[3] M. I. Dyakonov and V. I. Perel, ZhETF Pis. Red. 13, 657 (1971) [Sov. Phys. JETP. Lett. 13, 467 (1971)].

[4] J. E. Hirsch, Phys. Rev. Lett. 83, 1834 (1999).

[5] J. Sinova, D. Culcer, Q. Niu, N. A. Sinitsyn, T. Jungwirth, and A. H. MacDonald, Phys. Rev. Lett. 92, 126603 (2004).

[6] Y. K. Kato, R. C. Myers, A. C. Gossard, and D. D. Awschalom, Science 306, 1910 (2004).

[7] J. Wunderlich, B. Kaestner, J. Sinova, and T. Jungwirth, Phys. Rev. Lett. 94, 047204 (2005).

[8] S. O. Valenzuela and M. Tinkham, Nature (London) 442, 176 (2006).

[9] T. Kimura, Y. Otani, T. Sato, S. Takahashi, and S. Maekawa, Phys. Rev. Lett. 98, 156601 (2007).
[10] T. Seki, Y. Hasegawa, S. Mitani, S. Takahashi, H. Imamura, S. Maekawa, J. Nitta, and K. Takanashi, Nat. Mater. 7, 125 (2008).

[11] S. Takahashi and S. Maekawa, Sci. Technol. Adv. Mater. 9, 014105 (2008).

[12] E. Saitoh, M. Ueda, H. Miyajima, and G. Tatara, Appl. Phys. Lett. 88, 182509 (2006).

[13] S. Murakami, N. Nagaosa, and S.-C. Zhang, Science 301, 1348 (2003).

[14] J.-i. Inoue, G. E. W. Bauer, and L. W. Molenkamp, Phys. Rev. B 70, 041303 (2004).

[15] S. Zhang and Z. Yang, Phys. Rev. Lett. 94, 066602 (2005).

[16] W.-K. Tse and S. Das Sarma, Phys. Rev. Lett. 96, 056601 (2006). 
[17] P. L. Krotkov and S. Das Sarma, Phys. Rev. B 73, 195307 (2006).

[18] N. F. Mott and H. S. W. Massey, The Theory of Atomic Collisions (Oxford University Press, Oxford, 1949).

[19] J. Smit, Physica 24, 39 (1958).

[20] L. Berger, Phys. Rev. B 2, 4559 (1970).

[21] P. Nozières and C. Lewiner, J. Phys. (Paris) 34, 901 (1973).

[22] S. Zhang, Phys. Rev. Lett. 85, 393 (2000).

[23] X. Wang, J. Xiao, A. Manchon, and S. Maekawa, Phys. Rev. B 87, 081407 (2013).
[24] L. X. Hayden, R. Raimondi, M. E. Flatté, and G. Vignale, Phys. Rev. B 88, 075405 (2013).

[25] Z. Tešanović, M. V. Jarić, and S. Maekawa, Phys. Rev. Lett. 57, 2760 (1986).

[26] N. Trivedi and N. W. Ashcroft, Phys. Rev. B 38, 12298 (1988).

[27] N. W. Ashcroft and N. D. Mermin, Solid State Physics (Harcourt College Publishers, New York, 1985).

[28] C. Kittel, Introduction to Solid State Physics (Wiley, New York, 2005).

[29] X. Wang, J. Xiao, A. Manchon, and S. Maekawa, arXiv:1407.8278 [cond-mat]. 